\title{
A New ROI-Based performance evaluation method for image denoising using the Squared Eigenfunctions of the Schrödinger Operator
}

\author{
Abderrazak Chahid ${ }^{1}$, Hacene Serrai ${ }^{2}$, Eric Achten ${ }^{2}$, Taous-Meriem Laleg-Kirati ${ }^{1 \star}$ \\ ${ }^{1}$ Computer, Electrical and Mathematical Science and Engineering (CEMSE) division, \\ King Abdullah University of Sciences and Engineering (KAUST), KSA \\ ${ }^{2}$ University of Gent, Department of Radiology, Belgium
}

\begin{abstract}
In this paper, a new Region Of Interest (ROI) characterization for Magnetic Resonance Imaging (MRI) is proposed for denoising performance evaluation without noise estimation. This technique consists of balancing the contrast between the dark and bright ROIs to track the noise removal and achieve an optimal compromise between removing noise and preserving image details. The ROI technique has been tested using synthetic MRI images. It has been applied to a new denoising method called Semi-Classical Signal Analysis (SCSA).The SCSA decomposes the image into the squared eigenfunctions of the Schrödinger operator where a soft threshold $h$ is used to remove the noise from the noisy image. The obtained results using real MRI data prove that this method is suitable for real medical image processing where the noise-free image is not available.
\end{abstract}

Keywords: regions of interest (ROI), MRI, image enhancement, semi-classical signal analysis, Schrödinger Operator.

\section{INTRODUCTION}

Magnetic Resonance (MR) image enhancement is one of the growing and challenging research area due to its wide utilization for many clinical diagnosis processes and treatments. Despite the recent acquisition techniques such as Generalized Autocalibrating Partially Parallel Acquisition (GRAPPA), MRI is still a relatively long process that produces images with different levels of noise depending on the acquisition parameters and spatial resolution. Many research works have been conducted treating MRI image denoising from diverse perspectives which have been classified in different denoising categories [1] [2], for instance Non-Local Mean (NLM) based method [3] [4] [5], wavelet based method [6] [7] and the statistical approaches [8]. However, most of the state-of-art MRI denoising methods need insights about noise, by estimating noise distribution...etc., to be able to deal with the noise efficiently. These methods use statistical parameters such as Mean Squared Error (MSE) and Peak Signal-to-Noise Ratio (PSNR) and the Structural SIMilarity index (SSIM) [9] to evaluate their performances. However for real MR dataset, noise filtering and denoising performance evaluation are still some of the biggest challenges in MR . This is due to non-availability of the free noise images and the stronge correlation between the signal an noise. One of the very early studies that have been conducted to show the relationship between the measured contaminated signal has been presented by R.M Henkelman in [10]. In this work, the author has presented a correction factor, based on a background region, that must be subtracted from the measured signal to cancel noise. Moreover, to estimate the Signal-to-Noise Ratio (SNR), many other methods have been proposed based on the Region of Interest (ROI). Some approaches are based on the phased array magnitude images [11], the direct measurement of pixel SNR using repeated measurements for phantoms using both root-sum-of-squares (RSS) magnitude combined imaging and SENSE accelerated parallel imaging [12] or assessing the spatial SNR variations in dynamic contrast-enhanced (DCE) with different acquisition protocols [13]. In this paper, a new post-processing ROI-based technique has been developed to assess the denoising performance. The proposed metric is based on observing the standard deviation of an automatically selected ROIs in the image to evaluate the denoising performance. This metric is more suitable for iterative methods that don't estimate or use a predefined model for the noise. Therefore, The new metric has been used with a recent method so-called Semi-Classical Signal Analysis (SCSA) [14]. The later presents a new concept of image denoising based on the Schrödinger operator. Its denoising performance depends on the choice of a parameter called $h$ which needs an iterative search for the optimal value, using the proposed metric, to acheive the best possible denoising.

This paper is organized as follows. Section II introduces the proposed ROI-based method, the threshold setting, and the used algorithm to find an optimal $h$ for the SCSA method. Section III presents the results of the proposed method using real MRI images; a general conclusion is given in section IV.

\section{MATERIAL AND Methods}

\section{A. The proposed ROI-based evaluation metric}

Denoising performance evaluation is one of the most significant challenges for real image enhancement. As the reference free noise image in unknown, the developers use either visual evaluation to evaluate the denoised images or estimate the noise using filter of optimization based methods. In this paper, a new ROI-based method has been presented as a new solution for iterative methods that need to evaluate the denoising performance in each iteration to 


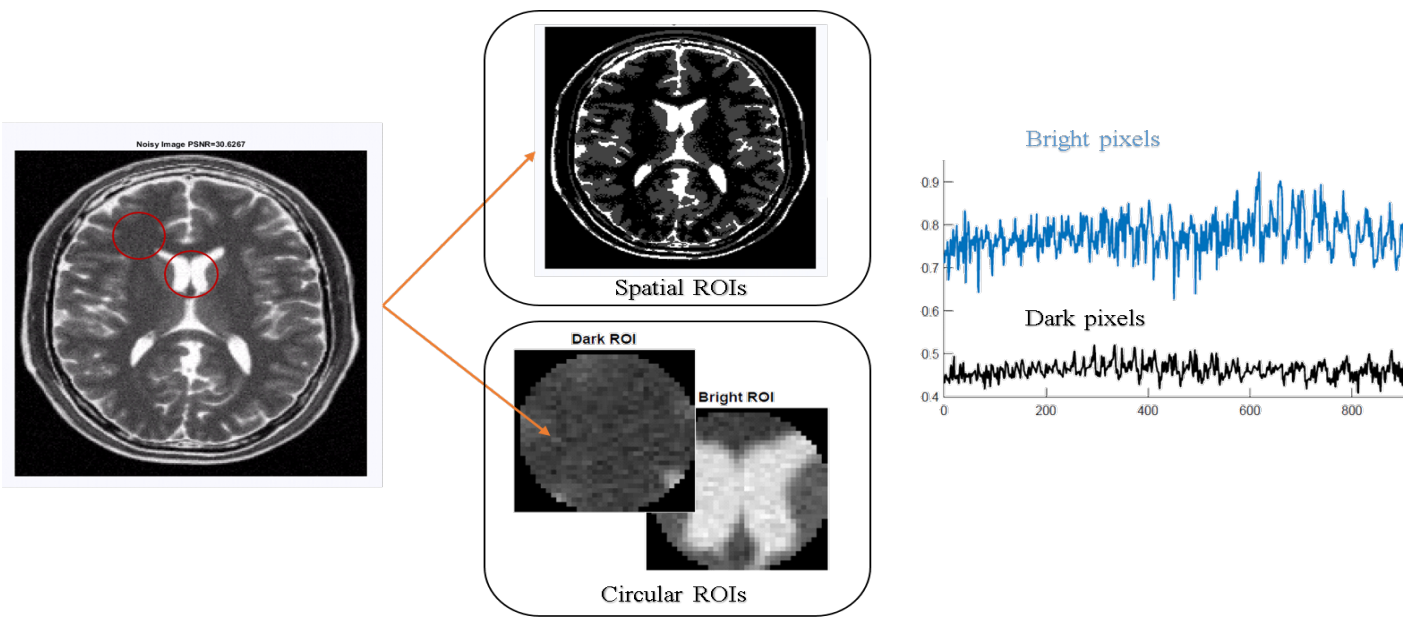

Fig. 1. The ROI-based method using circular and spatial Dark and Bright ROIs.

acheive better performance. The proposed ROI technique suggests focusing on the bright and dark regions of interest (ROI) to assess the denoising. These ROIs parts of the image are selected to be homogeneous tissues and away from regions with motion artifact or any irregular texture. In these two regions, the noise removal will be depend on the contrast and the smoothness of selected regions as shown in figure 1. The best performance is defined with the lower possible standard variation in both homogeneous bright and dark ROIs simultaneously. The mathematical representation of the proposed metrics that will be presented in the next section.

1) The circular ROI metric: For images that have large homogeneous dark and bright areas, a circular ROIs should be selected carefully to locate the area where the noise is concentrated. The amount of noise in the image is proportional to the standard deviation of these dark and Bright ROIs in the residual image. The more noise gets removed from the image the smoother and with lower standard deviation $\sigma$ in the selected ROIs. The new ROI-based denoising evaluation metric $S N R_{R O I}$ is defined as follows:

$$
\begin{aligned}
& S N R D R_{R O I}=\frac{\operatorname{Max}\left(I_{\text {Res }}\right)}{\sigma_{\text {Dark }}} \\
& S N R B D R R_{R O I}=\frac{\sigma_{D a r k}}{\sigma_{\text {Bright }}}
\end{aligned}
$$

where $\sigma_{\text {Dark }}$ and $\sigma_{\text {Bright }}$ are the standard deviation of the dark and bright ROIs of the residual image $I_{\text {Res }}$ respectively. and $I_{\text {Res }}=\left|I_{\text {Noisy }}-I_{\text {Denoised }}\right| . I_{\text {Noisy }}$ and $I_{\text {Denoised }}$ are the noisy and the denoised image.

2) The Spatial ROI metric: The circular ROI metric depends on the location and the size of the selected area as shown in figure 2 . Therefore, it gives only local information about the noise. Moreover, the metrics are not concave function which might misguide the iterative search of the optimal denoting performance. To solve this issues, all bight and dark parts in the image are concatenated to form a spatial regions that reflect the noise across the whole image.
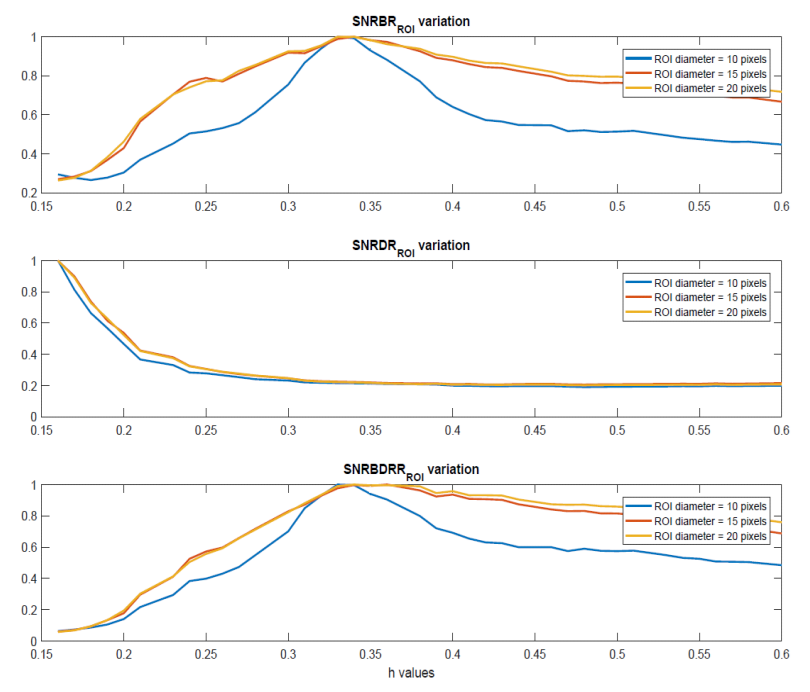

Fig. 2. Circular metrics VS ROI size.

This strategy is based on a threshold $\epsilon$ which defines the bright and dark pixels to be selected across the image. The pixel $I(x, y)$ in the image $I$ classified as follows:

$$
I(x, y) \in \begin{cases}\text { Bright } & 1-\epsilon \leq I(x, y) \leq 1 \\ \text { Dark } & 0 \leq I(x, y) \leq \epsilon\end{cases}
$$

The spatial evaluation metric $S N R_{R O I}$ using a given threshold $\epsilon$ is defined as follows:

$$
S N R_{R O I}(I)=20 \log \left(\frac{\operatorname{Max}\left(I_{R O I 0}\right)}{A_{0} \sigma_{D a r k}} \frac{\operatorname{Max}\left(I_{R O I 1}\right)}{A_{1} \sigma_{B r i g h t}}\right)
$$

where $\sigma_{\text {Dark }}$ ( resp. $\sigma_{\text {Bright }}$ ) is the standard deviation of the dark region ( resp. bright region) $I_{R O I 0}$ ( resp. $I_{R O I 1}$ ) which contains $A_{0}$ ( resp. $A_{0}$ ) number of pixels. 


\section{B. The spatial ROI metric validation}

The $S N R_{R O I}$ has been validated using synthetic data from BrainWeb database ${ }^{1}$. The $S N R_{R O I}$ has been applied to images with different levels of added Gaussian noise of variance $\sigma^{2}$. The figure 3 shows that the threshold $\epsilon=0.1$ gives the best noise tracking comparing the Peak Signal to Noise (PSNR).The spatial ROI-based metric shows higher ability to track the noise and prevents removing detail from the image or over smoothing it.
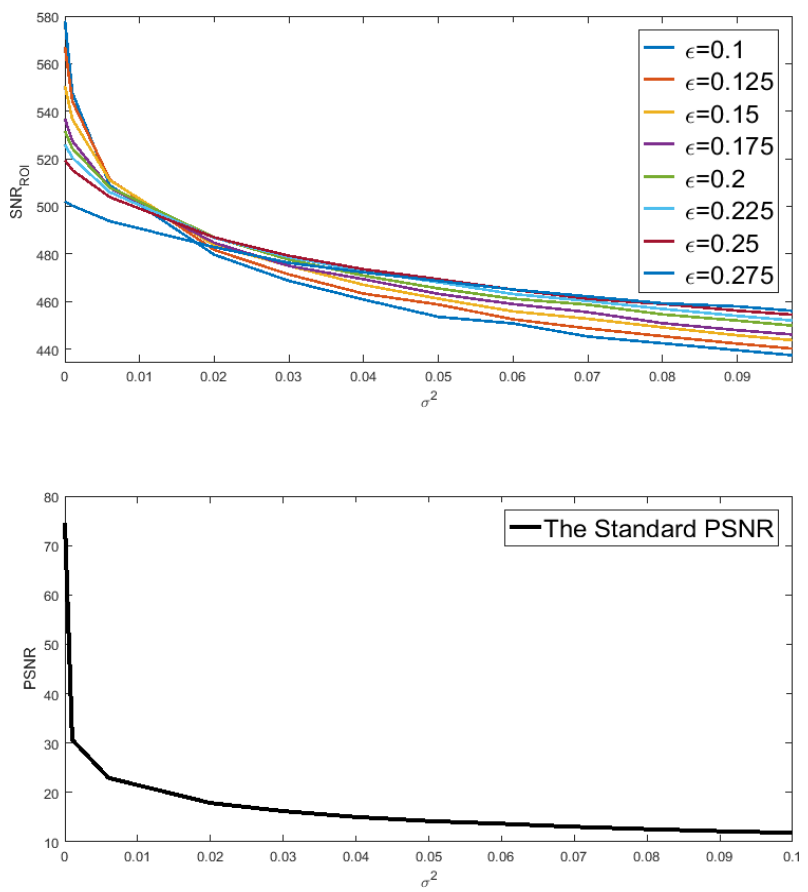

Fig. 3. $P S N R$ and $S N R_{R O I}$ comparison for noisy images using added Gaussian noise of variance $\sigma^{2}$.

\section{SCSA Denoising Method}

An efficient numerical algorithm has been proposed in [14] to reconstruct images using the SCSA. The idea consists in splitting the $2 \mathrm{D}$ Schrödinger operator $\mathscr{H}_{2, h}(I)$ into two unidimensional operators and in solving the eigenvalues problem row by row and column by column. The potential of the semi-classical Schrödinger operator is $x^{\text {th }}$ row and the $y^{t h}$ column of the noisy image $I$. The pixel $I_{h}(x, y)$ in the denoised image $I_{h}$ using the parameter value $h$ is reconstructed as follows:

$$
\begin{aligned}
I_{h}(x, y)= & \left(\frac{h^{2}}{L_{2, \gamma}^{c l}} \sum_{n=1}^{N_{n}} \sum_{m=1}^{M_{h}}\left(-\mu_{h}(n)+\rho_{h}(m)\right)^{\gamma}\right. \\
& \left.\cdot \psi_{n, h}^{2}(x) \cdot \phi_{m, h}^{2}(y)\right)^{\frac{1}{1+\gamma}}
\end{aligned}
$$

where $\gamma$ is a positive parameter that we choose equal to 4 and $L_{2, \gamma}^{c l}=\frac{1}{2^{2} \pi} \frac{\Gamma(\gamma+1)}{\Gamma(\gamma+2)}$. $\Gamma$ refers to the standard Gamma function. Moreover, $N_{h}$ ( resp. $M_{h}$ ) is the number of negative

\footnotetext{
${ }^{1}$ http://brainweb.bic.mni.mcgill.ca/brainweb/
}

eigenvalues $\mu_{h}(n)$ ( resp. $\rho_{h}(m)$ ) and the associated $L^{2}$ normalized eigenfunctions $\psi_{n, h}(x)$ ( resp. $\phi_{m, h}(y)$ ) of the one dimensional Schrödinger operator $\mathscr{H}_{x, h}\left(\right.$ resp. $\left.\mathscr{H}_{y, h}\right)$ such that:

$$
\begin{aligned}
& \mathscr{H}_{1, h}(I) \psi_{h}(x)=\mu_{h} \psi_{h}(x) \\
& \mathscr{H}_{1, h}(I) \phi_{h}(y)=\rho_{h} \phi_{h}(y)
\end{aligned}
$$

where

$$
\begin{aligned}
& \mathscr{H}_{2, h}(I)=-h^{2} \frac{\partial^{2}}{\partial x^{2}}+\frac{\partial^{2}}{\partial y^{2}}-I \\
& \mathscr{H}_{x, h}(I)=-h^{2} \frac{\partial^{2}}{\partial x^{2}}-I[x,:] \\
& \mathscr{H}_{y, h}(I)=-h^{2} \frac{\partial^{2}}{\partial y^{2}}-I[:, y]
\end{aligned}
$$

This method has been successfully applied for MRI denoising [15] where the soft threshold reflected by an appropriate choice of the parameter $h$ is used. The choice of this parameter is related to a compromise between accuracy and noise removal. Indeed, with larger $h$ values, more noise is removed but with more image blurring due to the smoothing of the image details.
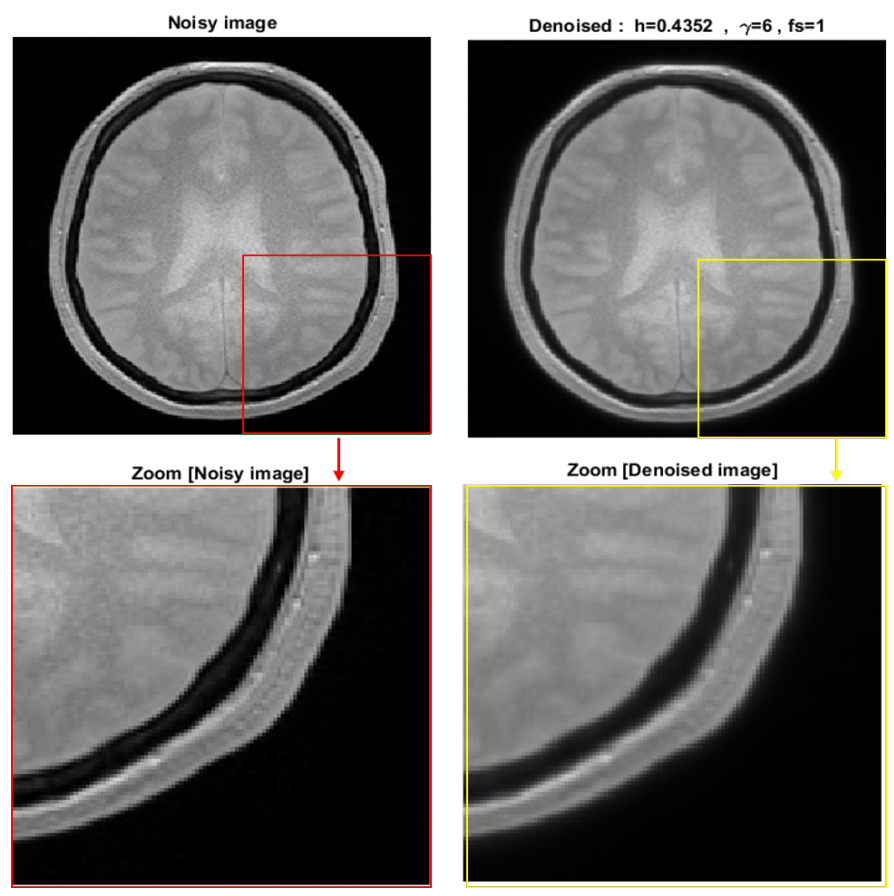

Fig. 4. Example1 of a denoised real MRI image.

\section{Results AND Discussion}

Experiments are performed on one healthy male subject, on a 3T scanner (MAGNETOM Tim-Trio, Siemens Healthcare) equipped with a 32-channel head coil for signal reception. Turbo Flash sequence is used with the following parameters: $\mathrm{TR} / \mathrm{TE}=250 / 2.46 \mathrm{~ms}$; matrix size, $256 \times 256$ resolution and 33 contiguous slices; FOV: 220mm; voxel size: $0.9 \times 0.9 \times 3 \mathrm{~mm} 3$; flip angle:10; and receiver bandwidth set to $320 \mathrm{~Hz} /$ pixel. The sets of data are acquired with Parallel Imaging (PI). 

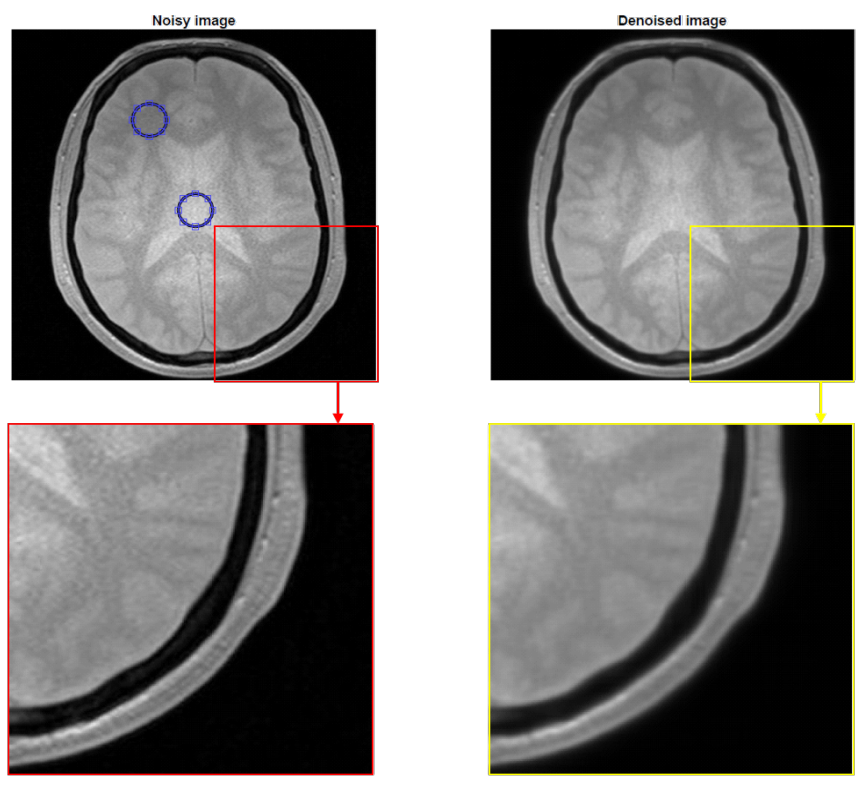

Fig. 5. Example2 of a denoised real MRI image.

The image denoising performance has been evaluated using two standard metrics: the Structural SIMilarity index (SSIM) [9] which evaluates the preservation of image details, the Global Phase Coherence (GPC) to measure the sharpness of the image [16]. The obtained results are shown in table I.

\begin{tabular}{|l|c|c|c|c|c|c|}
\cline { 2 - 7 } \multicolumn{1}{c|}{} & \multicolumn{3}{c|}{ Noisy image } & \multicolumn{3}{c|}{ Denoised image } \\
\hline Metrics & SSIM & SI & prop & SSIM & SI & prop \\
\hline image 1 & 0.9 & 506 & -506 & 0.85 & 239 & -564 \\
\hline Image 2 & 0.82 & 574 & -588 & 0.81 & 296 & -590 \\
\hline
\end{tabular}

TABLE I

THE EVALUATION METRIC RESULTS

The proposed metric $S N R_{R O I}$ was used as a convergence criterion to select the optimal parameter $h$. This range has been validated by visual investigations based on a set of real MRI denoised images.

The results in figure 4 and 5 and table I show that the proposed method was very helpful and constructive in guiding the SCSA method to achieve good MRI image enhancement as well as preserving details without the noisefree image.

\section{CONCLUSION}

In this paper, a new ROI based technique has been proposed for performance evaluation of MRI iterative denoising methods. It presents an efficient tool for denoising evaluation for real MRI dataset where the reference image is not available. This metric has been applied to a new MRI image enhancement method called SCSA. However, the noise in the real images is not constant. This can be explained by the fact that the SCSA algorithm may give be more efficient if the parameter $h$ would be adaptive to noise level and the image texture. In the future work, the adaptive SCSA will be compared to the most relevant MRI denoising methods. Finally, the presented utilization of bright and dark ROIs technique for image noise measurement will contribute to MRI image denoising especially helping developers to validate their algorithms for real dataset without a noiseless image.

\section{REFERENCES}

[1] J. Mohan, V. Krishnaveni, and Yanhui Guo, "A survey on the magnetic resonance image denoising methods," Biomedical Signal Processing and Control, vol. 9, pp. 56 - 69, 2014.

[2] J. Mohan, V. Krishnaveni, and Yanhui Guo, "A survey on the magnetic resonance image denoising methods," Biomedical Signal Processing and Control, vol. 9, pp. 56 - 69, 2014.

[3] José V Manjón, José Carbonell-Caballero, Juan J Lull, Gracián GarcíaMartí, Luís Martí-Bonmatí, and Montserrat Robles, "MRI denoising using non-local means," Medical image analysis, vol. 12, no. 4, pp. 514-523, 2008.

[4] Jun Jin, Elizabeth McKenzie, Zhaoyang Fan, Richard Tuli, Zixin Deng, Jianing Pang, Benedick Fraass, Debiao Li, Howard Sandler, Guang Yang, et al., "Nonlocal means denoising of self-gated and kspace sorted 4-dimensional magnetic resonance imaging using blockmatching and 3-dimensional filtering: Implications for pancreatic tumor registration and segmentation.," International journal of radiation oncology, biology, physics, vol. 95, no. 3, pp. 1058, 2016.

[5] Jinrong $\mathrm{Hu}$, Jiliu Zhou, and Xi Wu, "Non-local MRI denoising using random sampling," Magnetic Resonance Imaging, vol. 34, no. 7, pp. $990-999,2016$

[6] C. S. Anand and J. S. Sahambi, "MRI denoising using bilateral filter in redundant wavelet domain," in TENCON 2008 - 2008 IEEE Region 10 Conference, Nov 2008, pp. 1-6.

[7] Saime Akdemir Akar, "Determination of optimal parameters for bilateral filter in brain MR image denoising," Applied Soft Computing, vol. 43, pp. $87-96,2016$.

[8] Pierrick Coupé, José V. Manjón, Elias Gedamu, Douglas Arnold, Montserrat Robles, and D. Louis Collins, An Object-Based Method for Rician Noise Estimation in MR Images, pp. 601-608, Springer Berlin Heidelberg, Berlin, Heidelberg, 2009.

[9] Z. Wang, E. P. Simoncelli, and A. C. Bovik, "Multiscale structural similarity for image quality assessment," in Signals, Systems and Computers, 2004. Conference Record of the Thirty-Seventh Asilomar Conference on, Nov 2003, vol. 2, pp. 1398-1402 Vol.2.

[10] R.M. Henkelman, "Measurement of signal intensities in the presence of noise in mr images," Medical Physics, vol. 12, no. 2, pp. 232-233, 1985, cited By 709.

[11] Chris D Constantinides, Ergin Atalar, and Elliot R McVeigh, "Signalto-noise measurements in magnitude images from nmr phased arrays," Magnetic Resonance in Medicine, vol. 38, no. 5, pp. 852-857, 1997.

[12] Peter Kellman and Elliot R McVeigh, "Image reconstruction in snr units: a general method for snr measurement," Magnetic resonance in medicine, vol. 54, no. 6, pp. 1439-1447, 2005.

[13] Evanthia Kousi, Marco Borri, Jamie Dean, Rafal Panek, Erica Scurr, Martin O Leach, and Maria A Schmidt, "Quality assurance in mri breast screening: comparing signal-to-noise ratio in dynamic contrastenhanced imaging protocols," Physics in medicine and biology, vol. 61, no. 1, pp. 37, 2015.

[14] Z. Kaisserli, T.M. Laleg-Kirati, and A. Lahmar-Benbernou, "A novel algorithm for image representation using discrete spectrum of the Schrödinger operator," Digit. Signal Process., vol. 40, no. C, pp. 8087, May 2015.

[15] J. Zhang, T.M Laleg-Kirati, S. Bogaert, E. Achten, and H. Serrai, "Magnetic resonance imaging de-noising using the squared eigenfunctions of the Schrödinger operator: Application to brain MRI data," ISMRM 24th Annual Meeting, Singapore 2016., 2016.

[16] G. Blanchet and L. Moisan, "An explicit sharpness index related to global phase coherence," in 2012 IEEE International Conference on Acoustics, Speech and Signal Processing (ICASSP), March 2012, pp. 1065-1068. 